\title{
List of cases of the Court of Justice of the European Union
}

\section{Note: Electronic Reports of Cases (ECLI) numbers are given for all cases filed in 2010 or after.}

C-78/70 Grammophon Gesellschaft mbH v Metro-SB-Großmärkte GmbH \& Co. KG [1970] ECR 487

C-21/76 Handelskwekerij G. J. Bier BV v Mines de potasse d'Alsace SA [1978] ECR 1735

C-120/78 Rewe-Zentral AG v Bundesmonopolverwaltung für Branntwein [1979] ECR 649

C-34/79 R v Maurice Donald Henn and John Frederick Ernest Derby [1979] ECR 3795

C-76/90 Manfred Säger v Dennemeyer \& Co. Ltd [1991] ECR I-4221

C-68/93 Fiona Shevill, Ixora Trading Inc., Chequepoint SARL and Chequepoint International Ltd v Presse Alliance SA [1995] ECR I-415

C-55/94 Reinhard Gebhard v Consiglio dell'Ordine degli Avvocati e Procuratori di Milano [1995] ECR I-4165

C-53/96 Hermès International (a partnership limited by shares) v FHT Marketing Choice BV [1998] ECR I-03603

C-210/96 Gut Springenheide GmbH and Rudolf Tusky v Oberkreisdirektor des Kreises Steinfurt - Amt für Lebensmittelüberwachung [1998] ECR I-046571

C-440/97 GIE Groupe Concorde and others v Master of the Vessel Suhadiwarno Panjan and others [1999] ECR 6308

C-376/98 Federal Republic of Germany $v$ European Parliament and Council of the European Union [2000] ECR I-08419

C-381/98 Ingmar GB Ltd v Eaton Leonard Technologies Inc. [2000] ECR I-9305 118

C-101/01 Criminal Proceedings against Bodil Lindqvist [2003] ECR I-1297 298,

C-243/01 Criminal Proceedings against Piergiorgio Gambelli and others [2003] ECR I-13031

C-322/01 Deutscher Apothekerverband eV v 0800 Doc Morris NV and Jacques Waterval [2003] ECR I-14887

C-116/02 Erich Gasser GmbH v MISAT Srl [2003] ECR I-14693

C-203/02 British Horseracing Board Ltd and others $v$ William Hill Organisation Ltd [2004] ECR I-10415

C-237/02 Freiburger Kommunalbauten GmbH Baugesellschaft \& Co. KG v Ludger Hofstetter and Ulrike Hofstetter [2004] ECR I-03403

C-176/03 Commission of the European Communities $v$ Council of the European Union [2005] ECR I-7879 
C-168/05 Elisa María Mostaza Claro v Centro Móvil Milenium SL [2006] ECR I-10421

C-317/04 and C-318/04 European Parliament $v$ Council of the European Union and Commission of the European Communities [2006] ECR I-4721

12,327

C-4/03 Gesellschaft für Antriebstechnik $m b H \&$ Co. KG v Lamellen und Kupplungsbau Beteiligungs $K G(G A T)$ [2006] ECR I-6509

C-275/06 Productores de Música de España (Promusicae) v Telefónica de España SAU [2008] ECR I-271

179,236

C-533/07 Falco Privatstiftung and Thomas Rabitsch v Gisela Weller-Lindhorst [2009] ECR I-03327

C-557/07 LSG-Gesellschaft zur Wahrnehmung von Leistungsschutzrechten v Tele2 Telecommunication GmbH [2009] ECR I-01227

160,169

C-5/08 Infopaq International A/S v Danske Daglades Forening [2009] ECR I-6569 196

C-236/08 and C-238/08 Google France SARL and Google Inc. v Louis Vuitton Malletier SA and others [2010] ECR I-02417

176, 245

C-144/09 and C-585/08 Hotel Alpenhof GESMBH v Oliver Heller; Peter Pammer v Reederie Karl Schlüter GMBH \& Co. KG [2010] ECR I-12527

100, 110, 287

C-540/08, C-304/08, C-261/07 and C-299/07 Mediaprint; Zentrale zur Bekämpfung unlauteren Wettbewerbs eV; Galatea BVBA v Sanoma Magazines Belgium NV [2010] ECR I-10909

C-467/08 Padawan SL v Sociedad General de Autores y Editores de España (SGAE) [2010] ECR I-10055

C-19/09 Wood Floor Solutions and Andreas Domberger GmbH v Silva Trade SA [2010] ECR I-02121

C-403/08 and C-429/08 Football Association Premier League Ltd and others v QC Leisure and others; Karen Murphy v Media Protection Services Ltd [2011] ECR I-090834

197,252

C-323/09 Interflora and others $v$ Marks \& Spencer [2011] ECR I-08625 246

C-324/09 L'Oréal SA, Lancôme parfums et beauté \& Cie SNC, Laboratoire Garnier \& Cie and L'Oréal (UK) Ltd $v$ eBay International AG and others [2011] ECR I-06011

137, 176, 236, 245

C-393/09 Bezpečnostni softwarová asociace - Svaz softwarové ochrany v Ministry of Culture of the Czech Republic, ECLI:EU:C:2010:816, 22 December 2010

C-122/10 Konsumentombudsmannen v Ving Sverige AB, ECLI:EU:C:2011:299, 12 May 2011

C-509/09 and C-161/10 eDate Advertising GmbH v X; Olivier Martinez and Robert Martinez v MGN Ltd., ECLI:EU:C:2011:685, 25 October 2011

$67,95,147$

C-70/10 Scarlet Extended SA v Société belge des auteurs, compositeurs et éditeurs SCRL (SABAM), ECLI:EU:C:2011:771, 24 November 2011

184,301

C-604/10 Football Dataco Ltd v Yahoo! UK Ltd, ECLI:EU:C:2012:115, 1 March 2012

C-292/10 G v Cornelius de Visser, ECLI:EU:C:2012:142, 15 March $2012 \quad \mathbf{6 8 , 9 2}$

C-461/10 Bonnier Audio AB v Perfect Communication Sweden AB, ECLI:EU:C:2012: 219, 19 April 2012

184

C-523/10 Wintersteiger AG v Products $4 U$ Sondermaschinenbau GmbH, ECLI:EU:C: 2012:220, 19 April 2012

102

C-406/10 SAS Institute Inc. $v$ World Programming Ltd, ECLI:EU:C:2012:259, 2 May 2012

242, 244

C-5/11 Criminal Proceedings against Titus Alexander Jochen Donner, ECLI:EU:C: 2012:370, 21 June 2012 
C-128/11 UsedSoft GmbH v Oracle International Corp., ECLI:EU:C:2012:407, 3 July 2012

C-173/11 Football Dataco Ltd and others $v$ Sportradar GmbH and Sportradar AG, ECLI:EU:C:2012:642, 18 October 2012

C-428/11 Purely Creative Ltd. and others v Office of Fair Trading, ECLI:EU:C:2012: 651, 18 October 2012

280

C-607/11 ITV Broadcasting Ltd, ITV 2 Ltd, ITV Digital Channels Ltd, Channel 4 Television Corporation, 4 Ventures Ltd, Channel 5 Broadcasting Ltd and ITV Studios Ltd v TVCatchup Ltd, ECLI:EU:C:2013:147, 7 March 2013

C-657/11 Belgian Electronic Sorting Technology NV v Bert Peelaers, Visys NV, ECLI: EU:C:2013:516, 11 July 2013

C-170/12 Peter Pinckney v KDG Mediatech AG, ECLI:EU:C:2013:635, 3 October 2013

C-478/12 Armin Maletic and Marianne Maletic v lastminute.com GmbH and TUI Österreich GmbH, ECLI:EU:C:2013:735, 14 November 2013

C-281/12 Trento Sviluppo Srl and Centrale Adriatica Soc. Coop. Arl v Autorità Garante della Concorrenza e del Mercato, ECLI:EU:C:2013:859, 19 December 2013

C-355/12 Nintendo Co. Ltd, Nintendo of America Inc. and Nintendo of Europe GmbH v PC Box Srl and 9Net Srl, ECLI:EU:C:2014:25, 23 January 2014

C-466/12 Nils Svensson, Sten Sjögren, Madelaine Sahlman and Pia Gadd v Retriever Sverige $A B$, ECLI:EU:C:2014:76, 13 February 2014

C-351/12 OSA Ochranný svaz autorský pro práva k dílům hudebním os v Léčebné lázně Mariánské Lázně as, ECLI:EU:C:2014:110, 27 February 2014

C-314/12 UPC Telekabel Wien GmbH v Constantin Film Verleih GmbH and Wega Filmproduktionsgesellschaft mbH, ECLI:EU:C:2014:192, 27 March 2014 163,

C-387/12 Hi Hotel HCF Sarl v Uwe Spoering, ECLI:EU:C:2014:215, 3 April 2014100

C-342/13 Katalin Sebestyén v Zsolt Csaba Kövári and others, ECLI:EU:C:2014:1857, 3 April 2014

C-293/12 and C-594/12 Digital Rights Ireland Ltd $v$ Minister for Communications, Marine and Natural Resources, Minister for Justice, Equality and Law Reform, Commissioner of the Garda Siochána, Ireland and Attorney General, intervener: Irish Human Rights Commission; Kärntner Landesregierung, Michael Seitlinger, Christof Tschohl and others, ECLI:EU:C:2014:238, 8 April 2014

C-435/12 ACIAdam BV andothers vStichting de Thuiskopie, Stichting Onderhandelingen Thuiskopie vergoeding, ECLI:EU:C:2014:254, 10 April 2014

C-131/12 Google Spain SL and Google Inc. v Agencia Española de Protección de Datos (AEPD) and Mario Costeja González, ECLI:EU:C:2014:317, 13 May 2014

288, 318-20

C-360/13 Public Relations Consultants Association Ltd v Newspaper Licensing Agency Ltd and others, ECLI:EU:C:2014:1195, 5 June 2014

C-201/13 Johan Deckmyn and Vrijheidsfonds VZWv Helena Vandersteen, Christiane Vandersteen, Liliana Vandersteen, Isabelle Vandersteen, Rita Dupont, Amoras II CVOH and WPG Uitgevers België, ECLI:EU:C:2014:2132, 3 September 2014

C-291/13 Sotiris Papasavvas v O Fileleftheros Dimosia Etairia Ltd, Takis Kounnafi and Giorgos Sertis, ECLI:EU:C:2014:2209, 11 September 2014

54,170

C-117/13 Technische Universität Darmstadt v Eugen Ulmer KG, ECLI:EU:C:2014: 2196, 11 September 2014 
C-348/13 BestWater International GmbH v Michael Mebes and Stefan Potsch, ECLI: EU:C:2014:2315, 21 October 2014

C-441/13 Pez Hejduk v EnergieAgentur.NRW GmbH, ECLI:EU:C:2015:28, 22 January 2015

101

C-463/12 Copydan Båndkopi v Nokia Danmark A/S, ECLI:EU:C:2015:144, 5 March 2015

208

C-516/13 Dimensione Direct Sales Srl and Michele Labianca v Knoll International SpA, ECLI:EU:C:2015:315, 13 May 2015

205

C-322/14 Jaouad El Majdoub v CarsOnTheWeb.Deutschland GmbH, ECLI:EU:C: 2015:334, 21 May 2015

C-230/14 Weltimmo sr o v Nemzeti Adatvédelmi és Információszabadság Hatóság, ECLI:EU:C:2015:639, 1 October 2015

C-362/14 Maximillian Schrems v Data Protection Commissioner, joined party: Digital Rights Ireland Ltd, ECLI:EU:C:2015:650, 6 October 2015

C-347/14 New Media Online GmbH v Bundeskommunikationssenat, ECLI:EU:C:2015: 709, 21 October 2015

C-264/14 Skatteverket v David Hedqvist, ECLI:EU:C:2015:718, 22 October 2015

C-99/15 Christian Liffers $v$ Producciones Mandarina SL and Mediaset España Comunicación SA, formerly Gestevisión Telecinco SA, ECLI:EU:C:2016:173, 17 March 2016

C-57/15 United Video Properties, Inc. v Telenet NV, ECLI:EU:C:2016:611, 28 July 2016

C-160/15 GS Media BV v Sanoma Media Netherlands BV, Playboy Enterprises International Inc. and Britt Geertruida Dekker, ECLI:EU:C:2016:644, 8 September 2016

201

C-484/14 Tobias Mc Fadden v Sony Music Entertainment Germany GmbH, ECLI:EU: C:2016:689, 15 September 2016

C-582/14 Patrick Breyer v Bundesrepublik Deutschland, ECLI:EU:C:2016:779, 19 October 2016

C-203/15 and C-698/15 Tele2 Sverige AB v Post- och telestyrelsen and Secretary of State for the Home Department v Tom Watson and Others, ECLI:EU:C:2016:970, 21 December 2016

C-275/15 ITV Broadcasting Limited et al. v TVCatchup et al., ECLI:EU:C:2017:144, 1 March 2017

192,198

C-138/16 Staatlich genehmigte Gesellschaft der Autoren, Komponisten und Musikverleger registrierte Genossenschaft mbH (AKM) v Zürs.net Betriebs GmbH, ECLI:EU:C:2017:218, 16 March 2017

C-527/15 Stichting Brein v Jack Frederik Wullems (Filmspeler), ECLI:EU:C:2017: 300, 26 April 2017

C-610/15 Stichting Brein v Ziggo BV and XS4All Internet, BV, ECLI:EU:C:2017:456, 14 June 2017

C-194/16 Bolagsupplysningen OÜ, Ingrid Ilsjan v Svensk Handel AB, ECLI:EU:C: 2017:766, 17 October 2017

C-265/16 VCAST Limited v RTI SpA, ECLI:EU:C:2017:91, 29 November $2017 \mathbf{2 0 9}$

C-434/15 Asociación Profesional Élite Taxi v Uber Systems Spain, SL, ECLI:EU:C: 2017:232, 20 December 2017

C-498/16 Maximilian Schrems v Facebook Ireland Limited, ECLI:EU:C:2018:37, 25 January 2018 
C-210/16 Unabhängiges Landeszentrum für Datenschutz Schleswig-Holstein v Wirtschaftsakademie Schleswig-Holstein GmbH, ECLI:EU:C:2018:388, 5 June 2018

C-521/17 Coöperatieve Vereniging SNB-REACT U.A. v Deepak Mehta, ECLI:EU:C: 2018:639, 7 August 2018

C-161/17 Land Nordrhein-Westfalen v Dirk Renckhoff, ECLI:EU:C:2018:634, 7 August 2018

C-136/17 GC and Others v Commission nationale de l'informatique et des libertés (CNIL), ECLI:EU:C:2019:773, 4 October 2018

C-105/17 Komisia za zashtita na potrebitelite v Evelina Kamenova, ECLI:EU:C:2018: 808, 4 October 2018

C-149/17 Bastei Lübbe GmbH \& Co. KG v Michael Strotzer, ECLI:EU:C:2018:841, 18 October 2018

C-296/18 Conseil départemental de l'ordre des chirurgiens-dentistes de la Haute-Garonne $v R G$ and Dental Cabinet of Doctor RG SELARL, ECLI:EU:C:2018: 857, 23 October 2018

C-469/17 Funke Medien NRW GmbH v Bundesrepublik Deutschland, ECLI:EU:C: 2019:623, 16 January 2019

151-2, 221

C-142/18 Skype Communications Sàrl v Institut belge des services postaux et des télécommunications (IBPT), ECLI:EU:C:2019:460, 5 June $2019 \quad \mathbf{3 0 , 3 6}$

C-193/18 Google LLC v Bundesrepublik Deutschland, ECLI:EU:C:2019:498, 13 June 2019

31,36

C-649/17 Bundesverband der Verbraucherzentralen und Verbraucherverbände Verbraucherzentrale Bundesverband e.V. v Amazon EU Sàrl, ECLI:EU:C:2019:576, 10 July 2019

C-40/17 Fashion ID GmbH \& Co.KG v Verbraucherzentrale NRW eV, ECLI:EU:C: 2019:629, 29 July 2019

303

C-476/17 Pelham GmbH and Others v Ralf Hütter and Florian Schneider-Esleben, ECLI:EU:C:2019:624, 29 July 2019

152,222

C-516/17 Spiegel Online GmbH v Volker Beck, ECLI:EU:C:2019:625, 29 July 2019

152,222

C-299/17 VGMedia GesellschaftzurVerwertungderUrheber-undLeistungsschutzrechte von Medienunternehmen mbH v Google LLC, ECLI:EU:C:2019:716, 12 September 2019

228

C-507/17 Google LLC, successor in law to Google Inc. v Commission nationale de l'informatique et des libertés (CNIL), ECLI:EU:C:2019:772, 24 September 2019

C-673/17 Bundesverband der Verbraucherzentralen und Verbraucherverbände Verbraucherzentrale Bundesverband e.V. v Planet49 GmbH, ECLI:EU:C:2019:801, 1 October 2019

C-18/18 Eva Glawischnig-Piescze v Facebook Ireland Limited, ECLI:EU:C:2019:821, 3 October 2019

C-390/18 Airbnb Ireland, UC, ECLI:EU:C:2019:1112, 19 December 2019

C-263/18 Nederlands Uitgeversverbond and Groep Algemene Uitgevers v Tom Kabinet Internet BV, ECLI:EU:C:2019:1111, 19 December 2019

C-511/17 Györgyné Lintner v UniCredit Bank Hungary Zrt., ECLI:EU:C:2020:188, 11 March 2020

C-567/18 Coty Germany GmbH v Amazon Services Europe Sàrl, ECLI:EU:C:2020: 267, 2 April 2020 
C-311/18 Facebook Ireland and Schrems, ECLI:EU:C:2019:1145, 16 July $2020 \quad 327$

C-724/18 Cali Apartments SCI v Procureur général at the Cour d'appel de Paris, Ville de Paris, ECLI:EU:C:2020:743, 22 September 2020

C-623/17 C-511/18, C-512/18 and C-520/18 (joined cases) Privacy International $v$ Secretary of State for Foreign and Commonwealth Affairs et al., ECLI:EU:C:2020: 790,6 October 2020

C-682/18 LF v Google LLC, YouTube Inc., YouTube LLC, Google Germany GmbH [not yet decided]

C-401/19 Poland $v$ Parliament and Council [not yet decided]

C-500/19 Puls 4 TV GmbH \& Co. KG v YouTube LLC and Google Austria GmbH [not yet decided]

178

C-62/19 Star Taxi App SRL v Unitatea Administrativ Teritorială Municipiului București prin Primarul General and Consiliul General al Municipiului București, ECLI:EU: C:2020:692

T-873/16 Groupe Canal + v European Commission, ECLI:EU:T:2018:904, 12 December 2018

T-604/18 Google and Alphabet, ECLI:EU:T:2019:743, 23 September 2019 